\title{
Prophylactic laser treatment in early age related maculopathy reduced the incidence of exudative complications
}

\author{
C Frennesson, S E G Nilsson
}

\begin{abstract}
Aim-To investigate the effect of prophylactic laser treatment on drusen area and incidence of exudative lesions in patients with soft drusen maculopathy.

Methods-In a prospective study, patients with early age related maculopathy (ARM) and good visual acuity were randomised to laser treatment or to a control group. Each group consisted of two subgroups: a fellow eye group and a bilateral drusen group. At 3 years, 36 of 38 enrolled patients remained in the study. Photocoagulation was performed with an argon green laser, approximately 100 mild laser burns being placed on and between the drusen in a perifoveal temporal horseshoe-shaped area. Both cases and controls were subjected to fundus colour photographs and fluorescein angiograms at regular intervals, and the drusen area was calculated in both photographs and angiograms. At baseline, there were no significant differences $(p>0.3-0.8)$ in drusen area between the groups.
\end{abstract}

Results-In the treatment group, mean drusen area decreased significantly in the fundus photographs as well as in the angiograms $(p<0.001)$. Visual acuity and colour contrast sensitivity (CCS) did not change significantly. All these results are valid also for the subgroups. In the control group, however, mean drusen area increased significantly $(p<0.001)$. Mean visual acuity decreased significantly $(p<0.01)$ as did the colour contrast sensitivity along the tritan axis $(p=0.02)$. For the fellow eye control group $(n=7)$, the increase in drusen area in fundus photographs and the decrease in CCS along the tritan axis were not statistically significant $(p=0.57$ and $p=0.37$, respectively). Furthermore, at 3 years, five patients in the control group showed exudative lesions ( $1 / 7$ in the fellow eye group and 4/12 in the bilateral drusen group), whereas no such complications occurred in the treatment group. One patient developed a small atrophy, however. Thus, there is now a significant difference $(p=0.047)$, however with a large $95 \%$ confidence interval, 0.06-0.46, regarding exudative complications between the treated group and the control group in our small patient material.

Conclusion-Perifoveal mild laser treatment causes a reduction in drusen area in patients with soft drusen maculopathy and may lower the incidence of exudative lesions.

(Br f Ophthalmol 1998;82:1169-1174)

Soft drusen and hyperpigmentation are significant risk factors for the development of sight threatening lesions in age related macular degeneration (AMD) such as exudative complications - namely, choroidal neovascularisation (CNV) and pigment epithelial detachment (PED) or geographic atrophy. ${ }^{1-3}$ In previous studies, based either on populations or on patients attending ophthalmology departments, the cumulative risk incidence of sight threatening lesions for patients with bilateral drusen was estimated at $12 \%$ to $23 \%$ (3-5 years). ${ }^{134}$ Recently, in a hospital population of patients with bilateral drusen, the incidence rate of CNV was found to be as high as $25 \%$ within a follow up period of 2 years. ${ }^{5}$ Patients who had already lost one eye in CNV face a 3\% to $15 \%$ risk per year of developing exudative maculopathy in the other eye within the next 3-5 years. ${ }^{67}$ Patients presenting large drusen and focal hyperpigmentation in the other eye run a risk of $58 \%$ to $73 \%$ of losing central vision in the second eye within 5 years. ${ }^{28}$ Although multicentre clinical trials have shown a beneficial effect of laser treatment in selected cases, ${ }^{9}{ }^{10}$ only about $15 \%$ of patients with CNV are treatable under currently accepted guidelines ${ }^{11}$ and over $50 \%$ of the eyes treated will show recurrences. ${ }^{9}$ In the light of these circumstances, the need for alternative therapeutic modalities or, even better, prophylactic treatment, is obvious.

Several studies have reported that laser photocoagulation can cause a resolution of soft drusen, not only in treated areas but also in untreated areas. ${ }^{12-19}$ These observations provide the rationale for a prophylactic laser treatment in soft drusen maculopathy. However, the question arises as to whether the reduction in drusen area also reduces the long term risk of CNV. A prophylactic treatment should aim at more than resorbing drusen, the preferable aim being that of preventing exudative lesions.

In an ongoing prospective study, initiated in 1993, we have, so far, been able to demonstrate a significant reduction in drusen area as well as a significant decrease in exudative complications after prophylactic laser treatment of soft drusen.

Materials and methods

PATIENTS

Thirty eight patients with soft drusen maculopathy and a visual acuity of 0.8 or better were 
Table 1 Treatment group. Mean drusen area at study entry and at 3 years (SD)

\begin{tabular}{llll}
\hline & At study entry & At 3 years & p Value \\
\hline $\begin{array}{llll}\text { Combined group (n=17): } \\
\quad \text { Fundus photos }\end{array}$ & $7.2(4.7) \%$ & $2.1(1.9) \%$ & $<0.001$ \\
$\quad$ Angiograms & $17.7(9.9) \%$ & $9.6(7.2) \%$ & $<0.001$ \\
Fellow eye group (n=5): & $7.0(2.8) \%$ & $1.3(0.9) \%$ & 0.005 \\
$\quad$ Fundus photos & $16.0(11.9) \%$ & $11.6(10.4) \%$ & 0.035 \\
$\quad \begin{array}{ll}\text { Angiograms } \\
\text { Bilateral drusen group (n=12): }\end{array}$ & $7.3(5.5) \%$ & $2.5(2.1) \%$ & 0.01 \\
$\quad$ Fundus photos & $18.3(9.5) \%$ & $8.8(5.8) \%$ & $<0.001$ \\
$\quad$ Angiograms & & & \\
\hline
\end{tabular}

enrolled in the study. They were randomised to two equal groups: the treatment group and the control group. However, at 3 years, one man in the treatment group had died and one man was no longer able to participate as a result of a stroke. Subsequently, at 3 years the treatment group consisted of 17 patients, nine men and eight women. Five patients had a disciform lesion in the fellow eye and 12 patients had bilateral drusen. The better eye (that is, visual acuity of 0.8 or better) was chosen for treatment and in patients with good visual acuity in both eyes, one eye was randomised to treatment. Mean age of the treatment group was 71.6 (SD 6.5) years. The control group consisted of 19 patients, four men and 15 women. In this group, seven patients had a disciform lesion in the fellow eye and 12 had bilateral drusen. One eye was chosen to be included in the study using the same criteria as for the treatment group. Mean age of the control group was $68.5(6.2)$ years. There was no statistically significant difference in age between the two groups.

As stated above, the treatment group as well as the control group consisted of two subgroups, the fellow eye group, containing patients with exudative AMD in one eye and drusen maculopathy only in the other eye, and the bilateral drusen group, containing patients with drusen maculopathy in both eyes and no exudative changes in either eye. The mean age of the treated fellow eye group $(n=5)$ and of the bilateral drusen group $(\mathrm{n}=12)$ was 75.8 (3.6) years and 69.8 (6.6) years, respectively. The mean age of the fellow eye control group $(n=7)$ and of the bilateral drusen control group $(n=12)$ was $68.3(5.5)$ years and $68.5(6.8)$ years, respectively. At baseline, there was a significant difference in age between the two fellow eye groups, the treated group being older than the untreated group $(\mathrm{p}=0.02)$.

INCLUSION CRITERIA

All patients included in the study had soft drusen and visual acuity of 0.8 or better. They were allowed to have mild pigmentary changes.

Table 2 Control group. Mean drusen area at study entry and at 3 years (SD)

\begin{tabular}{|c|c|c|c|}
\hline & At study entry & At 3 years & $p$ Value \\
\hline \multicolumn{4}{|c|}{ Combined group $(n=19)$ : } \\
\hline Fundus photos & $8.3(5.7) \%$ & $11.5(7.7) \%$ & $<0.001$ \\
\hline Angiograms & $17.4(6.8) \%$ & $32.2(13.6) \%$ & $<0.001$ \\
\hline \multicolumn{4}{|c|}{ Fellow eye group $(n=7)$ : } \\
\hline Fundus photos & $5.7(3.4) \%$ & $6.9(3.6) \%$ & 0.57 \\
\hline Angiograms & $19.1(8.7) \%$ & $34.1(12.3) \%$ & 0.004 \\
\hline \multicolumn{4}{|c|}{ Bilateral drusen group $(n=12)$ : } \\
\hline Fundus photos & $9.8(6.3) \%$ & $13.8(8.3) \%$ & $<0.001$ \\
\hline Angiograms & $16.4(5.5) \%$ & $31.3(14.6) \%$ & 0.001 \\
\hline
\end{tabular}

Patients with more advanced changes such as pigmentary clumping, pigment epithelial detachment (PED), choroidal neovascularisation and haemorrhage or macular atrophy were excluded. This also applied to those who, apart from the drusen maculopathy, suffered from any other eye disorder which could possibly influence the result. No systemic risk factors were registered.

\section{EXAMINATION}

Both cases and controls were subjected to examinations including best corrected visual acuity, slit lamp examination of the anterior segment, and biomicroscopy of the posterior segment (dilated). Refraction was performed in a standardised fashion using a Snellen's letter chart (GP chart, Preisler, Malmö, Sweden) at a distance of 5 metres. The anterior segment, the optic disc, and the peripheral retina were normal. Intraocular pressure (Goldmann applanation) was less than $22 \mathrm{~mm}$ $\mathrm{Hg}$.

To test colour vision, the D-15 panel (Vitactive, Stockholm, Sweden) was used. Colour contrast sensitivity was assessed measuring protan, deutan, and tritan thresholds with a computer graphics system and procedure as previously described ${ }^{20-22}$ with some modifications. ${ }^{1522}$ The colours were displayed on an NEC Multi Sync 6 FG monitor with a dot pitch of $0.28 \mathrm{~mm}$ run by a $25 \mathrm{MHz}$ Intel 80386 DX computer containing a graphics card (TSL 206 with a 24 bit palette) and a software graphics program. At the resolution we used $(960 \times 760$ pixels $)$ the graphics card has a refresh rate of $90 \mathrm{~Hz}$.

The central visual field was evaluated using the Humphrey fields analyser 640 (Carl Zeiss AB, Stockholm, Sweden), 10-2 threshold test. To obtain the mean threshold value for the central field, the printout in decibels (dB) (logarithmic scale) was converted to apostilb (Asb) (linear scale).

\section{PHOTOGRAPHY AND ANGIOGRAPHY}

Standard fundus photographs $\left(50^{\circ}\right)$ and fluorescein angiograms were performed using a Nikon fundus camera NF-505 (Bergströms Instrument, Stockholm, Sweden).

The drusen area was evaluated from the fundus photographs and angiograms using a scanner and a computer program as previously described. ${ }^{15}$ The total drusen area is estimated as a part of the area of a circle, centred over the fovea, with a radius of $2500 \mu \mathrm{m}$ in the fundus photographs and $1250 \mu \mathrm{m}$ in the angiograms.

\section{FOLLOW UP}

Follow up visits were scheduled for cases and controls at 1 month after enrolment, every 3 months the first year, and then at 2, 2.5, and 3 years. Each time, the patients were examined as described above and fundus photography and 


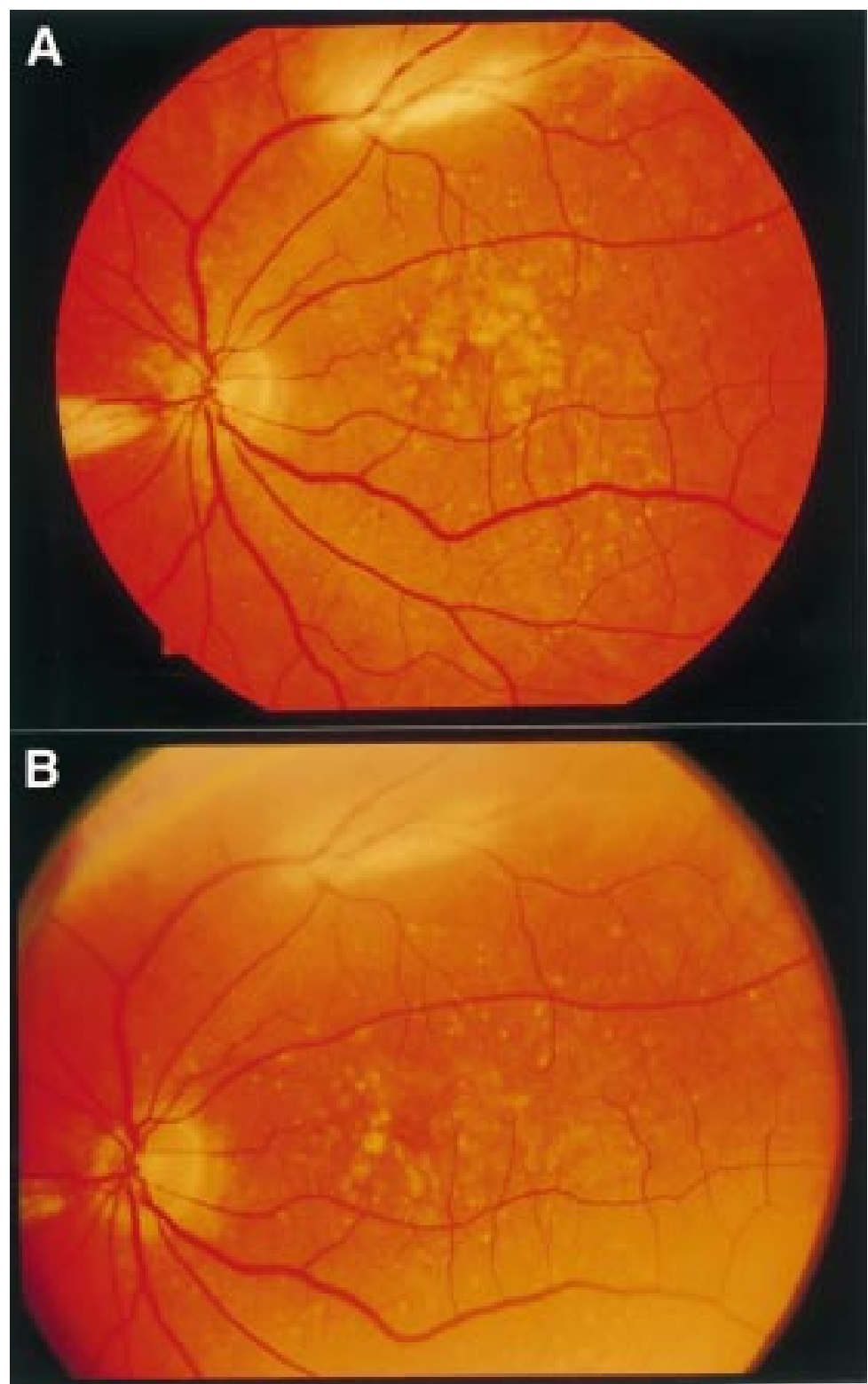

Figure 1 Colour fundus photographs of a 70 year old man (A) before and (B) 3 years after perifoveal laser photocoagulation.

angiography were performed (with the exception of 9 months post baseline).

\section{TREATMENT}

Photocoagulation was performed with an argon green laser (Lasertek $41 \mathrm{AK}$, Martinsson \& Co Instrument AB, Hägersten, Sweden). Approximately 100 laser burns (range 51-154) were placed as a direct treatment of drusen, rather than being placed in a specific pattern or as a grid, as well as scattered over drusen-free areas. No confluent laser burns were applied. The treatment area comprised a temporal horseshoe-shaped area extending to the vascular arcades. In about one third of the cases, the laser burns were placed no closer than approximately $500 \mu \mathrm{m}$ to the centre of the fovea. In the remaining cases, the laser burns were placed no closer than approximately $750 \mu \mathrm{m}$ to the centre of the fovea. Spot size was $200 \mu \mathrm{m}$, duration 0.05 seconds, and the power precisely sufficient to produce a barely visible lesion (0.1-0.2
W) which was the endpoint of treatment. No heavy white burns were applied.

The study was approved by the ethics committee of Linköping University and all subjects gave their informed consent before participation.

\section{STATISTICAL METHODS}

Student's $t$ test was used for comparing the treatment group and the control group and paired $t$ test for comparisons within the two groups. The two sided test was used to determine the $\mathrm{p}$ value. The $t$ test based on the pooled standard deviation was used to compare the change across the groups. To compare the rate of development of exudative complications in the two groups, we used Fisher's exact test.

\section{Results}

DRUSEN AREA

At baseline, there was no significant difference in mean drusen area between the treatment group and the control group, between the two fellow eye groups or between the two bilateral drusen groups, either in the fundus photographs or in the angiograms $(p>0.3-0.8)$. In the treatment group, the mean drusen area decreased significantly $(\mathrm{p}<0.001)$ when measured both in the fundus photographs and the angiograms. In both subgroups in the treatment group, the mean drusen area decreased significantly $(\mathrm{p}=0.03-<0.001)$ (Table 1, Fig 1). During the same time, the mean drusen area in the control group increased significantly $(p<0.001)$ in the fundus photographs and the angiograms (Table 2). However, for the fellow eye group $(n=7)$ the increase was statistically significant $(p=0.004)$ for the central part of the macula, as calculated in the angiograms, but not for the entire area (as determined in the fundus photographs) $(\mathrm{p}=0.57)$ (Table 2). Comparing the change in drusen area from baseline to 3 years for the two fellow eye groups, the differences were significant both for the fundus photographs and the angiograms $(p=0.003-<0.001)$. In the bilateral drusen group $(n=12)$, the increase was statistically significant ( $\mathrm{p}$ 0.001) for the fundus photographs as well as for the angiograms (Table $2)$. The change in drusen area across the two groups was also significant $(\mathrm{p}<0.001)$.

\section{VISUAL ACUITY}

Visual acuity was measured using a Snellen's chart and is reported in the Snellen's fraction (0.1-1.0) and not in log MAR (MAR = the reciprocal of the Snellen fraction).

Before treatment all patients had a visual acuity of $0.8-1.0$. In the treatment group, mean visual acuity did not change significantly $(p=0.70)$ from study entry to 3 years (Table 3$)$. Visual acuity improved in two patients by two lines, remained the same in 13 patients, and deteriorated by two lines in one patient. In one patient, visual acuity deteriorated by three lines as a consequence of a small atrophy. No change in either of the subgroups of the treatment group was noted (Table 3). In the control group, mean visual acuity decreased significantly $(p<0.01)$ from study entry to 3 years (Table 3). Visual acuity improved in one 
Table 3 Visual acuity at study entry and at 3 years (SD)

\begin{tabular}{llll}
\hline & At study entry & At 3 years & p Value \\
\hline Combined treatment group $(\mathrm{n}=17)$ & $0.94(0.09)$ & $0.92(0.14)$ & 0.70 \\
Fellow eye group $(\mathrm{n}=5)$ & $0.98(0.04)$ & $0.92(0.13)$ & 0.21 \\
Bilateral drusen group $(\mathrm{n}=12)$ & $0.92(0.09)$ & $0.93(0.15)$ & 0.83 \\
Combined control group $(\mathrm{n}=19)$ & $0.95(0.08)$ & $0.79(0.24)$ & $<0.01$ \\
Fellow eye group $(\mathrm{n}=7)$ & $0.97(0.08)$ & $0.78(0.37)$ & 0.26 \\
Bilateral drusen group $(\mathrm{n}=12)$ & $0.94(0.09)$ & $0.73(0.26)$ & 0.005 \\
\hline
\end{tabular}

Table 4 Colour contrast thresholds at study entry and at 3 years (SD)

\begin{tabular}{|c|c|c|c|}
\hline & Protan & Deutan & Tritan \\
\hline \multicolumn{4}{|c|}{ Combined treatment group $(n=17)$ : } \\
\hline At study entry & $12.6(9.3)$ & $15.2(11.5)$ & $28.3(21.1)$ \\
\hline At 3 years & $14.3(11.7)$ & $15.6(12.4)$ & $33.3(19.7)$ \\
\hline $\mathrm{p}$ Value & 0.37 & 0.84 & 0.15 \\
\hline \multicolumn{4}{|c|}{ Combined control group $(n=19)$ : } \\
\hline At study entry & $11.4(6.4)$ & $13.0(7.1)$ & $19.5(11.2)$ \\
\hline At 3 years & $13.8(8.2)$ & $14.4(10.0)$ & $28.7(16.7)$ \\
\hline $\mathrm{p}$ Value & 0.28 & 0.49 & 0.02 \\
\hline \multicolumn{4}{|c|}{ Fellow eye group $(n=7)$ : } \\
\hline At study entry & $11.1(4.6)$ & $13.4(5.2)$ & $19.4(8.5)$ \\
\hline At 3 years & $11.1(6.2)$ & $12.5(8.2)$ & $20.8(8.3)$ \\
\hline $\mathrm{p}$ Value & 0.55 & 0.96 & 0.37 \\
\hline \multicolumn{4}{|c|}{ Bilateral drusen group $(n=12)$ : } \\
\hline At study entry & $11.6(7.4)$ & $12.7(8.2)$ & $19.5(12.8)$ \\
\hline At 3 years & $15.1(8.9)$ & $15.3(11.0)$ & $32.7(18.6)$ \\
\hline $\mathrm{p}$ Value & 0.13 & 0.21 & 0.01 \\
\hline
\end{tabular}

patient by two lines, remained the same in 10 patients, and deteriorated by two lines in one patient and by three lines or more in seven patients, one of whom decreased to hand movements due to a $\mathrm{CNV}$ with a widespread haemorrhage. The bilateral drusen group $(n=12)$ of the control group showed a significant $(\mathrm{p}=0.005)$ decrease in visual acuity (Table 3). Mean visual acuity for the fellow eye group $(\mathrm{n}=7)$ decreased from 0.97 (SD 0.08) at study entry to $0.78(0.37)$ at 3 years $(p=0.26)$ (Table 3).

COLOUR CONTRAST SENSITIVITY

Colour contrast sensitivity for all three colour axes was low compared with an age matched group of normals. ${ }^{22}$ There were, however, no differences between the combined treatment group and the combined control group at study entry $(p>0.1-0.6)$. Comparing the two fellow eye groups at baseline, the treatment group showed a lower colour contrast sensitivity along the tritan axis than the control group $(p=0.04)$. No significant changes at 3 years were observed in the treatment group (Table 4). However, among the controls, the bilateral drusen group showed a significant decrease in mean colour contrast sensitivity along the tritan axis $(\mathrm{p}=0.01)$; the thresholds for the protan and deutan axes remaining unchanged (Table 4).

No differences were observed in either group regarding central visual field $(\mathrm{p}>0.07)$ or colour vision.

Table 5 Time of development of exudative complications in the control group

\begin{tabular}{lll}
\hline & $\begin{array}{l}\text { Fellow eye } \\
\text { group }\end{array}$ & $\begin{array}{l}\text { Bilateral drusen } \\
\text { group }\end{array}$ \\
\hline CNV: & & 36 months \\
Classic & 12 months (+PED) \\
Occult & 28 months \\
$\begin{array}{ll}\text { CNV with haemorrhage } \\
\text { PED }\end{array}$ & 12 months & 12 months \\
\hline
\end{tabular}

COMPLICATIONS

No patients in the treatment group suffered from exudative lesions. One patient, however, developed a small atrophy. In the control group, five patients developed exudative lesions during follow up (Table 5). Four patients showed choroidal neovascularisations: one classic CNV, two occult CNVs (one with a PED), and one CNV with a widespread haemorrhage. In the case of classic CNV, conventional photocoagulation was administered, and the patient with a fibrovascular membrane causing a haemorrhage was subjected to subfoveal surgery with no improvement in vision. A fifth patient developed a PED (Table 5). Of these five patients, only one, the patient with a CNV causing a severe haemorrhage, belonged to the high risk group with an exudative lesion in the fellow eye before enrolment. Thus, there is now a significant difference between the two groups regarding exudative lesions $(p=0.047)(95 \%$ confidence interval, 0.06-0.46).

\section{Discussion}

Gass reported in 1973 that laser photocoagulation could cause resolution of soft drusen ${ }^{23}$ and he was the first to suggest prophylactic treatment for AMD. His report has been followed by others, which differ to some extent in terms of laser technique, numbers of laser burns, and the time of intervention in the course of the disease. The present study, which was the first randomised and controlled study to be initiated, is in concordance with other surveys finding a significant reduction in drusen area after laser photocoagulation, not only of treated drusen but also of untreated foveal drusen. In our study, the resolution took place within the first 12 months, the largest changes occurring during the latter half of the period.

In the combined control group, mean visual acuity decreased significantly, as did colour contrast sensitivity for the tritan axis, indicating an impairment of retinal function. Mean visual acuity decreased in both subgroups. On the other hand, we found no improvement of mean visual acuity following treatment, as reported by others. ${ }^{17} 18{ }^{24}$ However, there was no appreciable visual loss before treatment, mean visual acuity being 0.94 (SD 0.09). The significant change in colour contrast sensitivity for the tritan axis was found only in the bilateral drusen group $(n=12)$, not in the fellow eye group $(n=7)$. At baseline, the fellow eye group in the treatment group $(n=5)$ had a lower colour contrast sensitivity along the tritan axis compared with the control fellow eye group $(n=7)(p=0.04)$. There was no significant change from baseline to 3 years for either of the groups $(p=0.07-0.8)$.

The fellow eye group of the treatment group had significantly higher age than the control fellow eye group $(p=0.02)$. This does not seem to favour the treatment group.

The aim of a prophylactic treatment ought to be more than mere resorption of drusennamely, the prevention of exudative complications. We found significantly more exudative 
complications in the control group $(5 / 19=$ $26 \%)$ than in the treatment group $(0 / 17)$ after a follow up period of 3 years. One of seven in the fellow eye group (14\%) and four of 12 $(33 \%)$ in the bilateral drusen group advanced to an exudative stage of the disease. This is markedly higher than expected. The 5 year cumulative incidence of late ARM in persons with bilateral early ARM in the Beaver Dam Eye Study was $11.7 \%$. Exudative lesions developed in $7.1 \% .^{3}$ In a study from Moorfields Eye Hospital, the 3 year cumulative incidence was $23.5 \%$ for late ARM and $18 \%$ for exudative AMD. ${ }^{4}$ However, it should be observed that, in our study, the confidence interval is large $(6 \%-$ $46 \%$ ), owing to the small number of patients, and therefore the magnitude of the difference in complication rate ought to be measured and evaluated with caution. No exudative lesions occurred in the treatment group, contrasting with previous studies, where the incidence of CNVs in treated eyes was $4-11 \% .^{12} 1317$ This divergence may be related to the number of laser burns, the intensity of the burns, the inclusion criteria - that is, how early the eyes are treated, and the sample size. Compared with most studies, we apply a fairly large number of laser burns, on average 100. Little et $a l^{18}$ recently published a controlled study, where 27 patients with symmetrical soft drusen maculopathy were randomised to prophylactic laser treatment (approximately 130 laser burns per eye) or to no treatment. After a follow up of 3.2 years, on average, two treated eyes had advanced to an exudative stage (two CNV), whereas 10 of the untreated eyes showed exudative complications (four CNV and six drusenoid PED). Thus, in agreement with our results, they found that progression to $\mathrm{CNV}$ was reduced by treatment. However, a multicentre, randomised clinical study in the USA (The Choroidal Neovascularisation Prevention Trial, CNVPT) found an increase of the incidence of $\mathrm{CNV}$ in treated eyes in the fellow eye group. ${ }^{25}$ Ten of 59 treated eyes developed CNV during the first 18 months, whereas only two of 61 observed eyes were affected $(p=0.02)$. In the bilateral drusen study, four of 156 treated eyes and two of 156 observed eyes developed CNV ( $p=0.62)$. Thus, according to this study, laser treatment may increase the short term incidence of CNV. Compared with our study, the CNVPT protocol differs in inclusion criteria (allowing patients with more advanced maculopathy (visual acuity $20 / 40$ or better, PED <1 disc diameter; geographic atrophy outside $500 \mu \mathrm{m}$ from the centre of the avascular zone) to enter), number and distribution of laser burns (20-24 burns placed in two to three rows temporal or in a circular pattern around the fovea). The optimal number of laser burns is disputed. Guymer et al ${ }^{19}$ recently showed that drusen disappeared following a few laser lesions, without any impending risk of CNV. Whereas small, intense laser burns may imply an increased risk of rupture of Bruch's membrane and $\mathrm{CNV},{ }^{26}$ mild laser photocoagulation is known to cause a proliferation of the retinal pigment epithelium (RPE).$^{27}{ }^{28}$ Mitosis, however, seems to be a local effect. ${ }^{29}$ There- fore, to obtain a favourable repopulation of the RPE, a fairly large number of laser burns may be required.

It seems important to apply the treatment at an early stage of the disease. Sarks et $a l^{17}$ conclude that the risk of complications may be reduced by treating eyes early, before the development of pigmentary changes. Outside the study, we treated patients who did not meet our inclusion criteria. In two cases, CNV developed. In both cases, the maculopathy was more advanced than in the patients participating in the study. The first case had a minor PED overlying a drusen at the time of treatment and the second case had a serous PED 6 years before treatment. CNV developed at 28 and 10 months, respectively.

The mechanism by which photocoagulation promotes the disappearance of drusen is not known. Mild laser photocoagulation itself can cause proliferation of the RPE. Furthermore, a diffusible factor may be involved since drusen disappear also at a distance from the photocoagulation sites.

Different growth factors have been shown in proliferating RPE cells and may play a role in wound repair after laser photocoagulation. ${ }^{30} 31$

The RPE phagocytoses shed photoreceptor outer segments throughout life, with a subsequent accumulation of lipofuscin. In order to increase the phagocytic capacity of the RPE, it may be beneficial to repopulate it. Mitosis in the RPE could reduce the amount of debris per cell, which may possibly enhance the capacity of also phagocytosing drusen material. It is also possible that laser treatment alters the permeability of Bruch's membrane and the metabolic activity of the RPE.

Processes from the choriocapillaris and pericytes have been proposed to play a role in the normal clearance of drusen material. ${ }^{32}{ }^{33}$ Laser photocoagulation caused an upregulation in the size and number of processes which, in turn, might explain the disappearance of drusen after such treatment.

We found not only a significant reduction in drusen area but also a decreased risk of exudative lesions following prophylactic photocoagulation of soft drusen. Our material is small, however, and conclusions must be drawn with some caution. A larger prospective, clinical trial is needed to determine the long term benefit of the treatment principles used in our study.

The present study was supported by grants from the Swedish Medical Research Council (Project No 12X-734), from the Research Committee of the County of Östergötland and from Synfrämjandet's Research Foundation.

1 Smiddy WE, Fine SL. Prognosis of patients with bilateral macular drusen. Ophthalmology 1984;91:271-7.

2 Bressler SB, Maguire MG, Bressler NM, et al, the Macular Photocoagulation Study Group. Relationship of drusen and abnormalities of the retinal pigment epithelium to the prognosis of neovascular macular degeneration. Arch Ophthalmol 1990;108:1442-7.

3 Klein R, Klein BEK, Jensen SC, et al. The five-year incidence and progression of age-related maculopathy. The Beaver Dam Eye Study. Ophthalmology 1997;104:7-21.

4 Holz FG, Wolfensberger TJ, Piguet B, et al. Bilateral macular drusen in age-related macular degeneration: prognosis and risk factors. Ophthalmology 1994;101:1522-8.

5 Sarraf D, Gin T, Brannon A, et al. Long-term drusen study: evaluation of incidence of choroidal neovascular membrane formation. Invest Ophthalmol Vis Sci 1997;38 (suppl 4):s18. 
6 Gregor Z, Bird AC, Chisholm IH. Senile disciform macular degeneration in the second eye. Br F Ophthalmol 1977;61: degene $141-7$.

7 Strahlman ER, Fine SL, Hillis A. The second eye of patients with senile macular degeneration. Arch Ophthalmol 1983; 101:1191-3.

8 Macular Photocoagulation Study Group. Risk factors for choroidal neovascularization in the second eye of patients with juxtafoveal or subfoveal choroidal neovascularization secondary to age-related macular degeneration. Arch Oph thalmol 1997;115:741-7.

9 Macular Photocoagulation Study Group. Argon laser photocoagulation for neovascular maculopathy. Five-year results from randomized clinical trials. Arch Ophthalmol 1991;109:1109-14

10 Macular Photocoagulation Study Group. Laser photocoagulation for juxtafoveal choroidal neovascularization: fiveyear results from randomized clinical trials. Arch Ophthalmol 1994;112:500-9.

11 Freund KB, Yanuzzi LA, Sorenson JA. Age-related macular degeneration and choroidal neovascularization. $A m ₹ \mathrm{Oph}$ degeneration and choroidal

12 Cleasby CW, Nakanishi AS, Norris LJ. Modern problems in ophthalmology. Basle: Karger:1979;20:141-7.

13 Wetzig PC. Photocoagulation of drusen-related macular degeneration: a long-term outcome. Trans Am Ophthalmo Soc 1994;92:299-306.

14 Figueroa MS, Regueras A, Bertrand J. Laser photocoagulation to treat macular soft drusen in age-related macula degeneration. Retina 1994;14:391-6.

15 Frennesson IC, Nilsson SEG. Effects of argon (green) laser treatment of soft drusen in early age-related maculopathy (ARM). A six-month prospective study. $\mathrm{Br} \mathcal{F}$ Ophthalmol 1995;79:905-9.

16 Frennesson IC, Nilsson SEG. Laser photocoagulation of soft drusen in early age-related maculopathy (ARM). The one-year results of a prospective, randomised trial. Eur $\mathcal{f}$ Ophthalmol 1996;6:307-14.

17 Sarks SH, Arnold JJ, Sarks JP, et al. Prophylactic perifoveal laser treatment of soft drusen. Aust NZ $\mathcal{F}$ Ophthalmol 1996; 24:15-26.

18 Little HL, Showman JM, Brown BW. A pilot randomized controlled study on the effect of laser photocoagulation of confluent soft drusen. Ophthalmology 1997;104:623-31.

19 Guymer RH, Gross-Jendroska M, Owens SL, et al. Laser treatment in subjects with high-risk clinical features of agerelated macular degeneration. Posterior pole appearance and retinal function. Arch Ophthalmol 1997;115:595-603.
20 Arden GB, Gündüz K, Perry S. Color vision testing with a computer graphics system. Clin Vis Sci 1988;2:303-20.

21 Arden GB, Berninger T, Hogg C, et al. A survey of color discrimination in German ophthalmologists. Changes associated with the use of lasers and operating microscopes. Ophthalmology 1991;98:567-75.

22 Frennesson IC, Nilsson UL, Nilsson SEG Colour contrast sensitivity in patients with soft drusen, an early stage of ARM. Doc Ophthalmol 1995;90:377-86.

23 Gass JMD. Drusen and disciform macular detachment and degeneration. Arch Ophthalmol 1973;90:206-17.

24 Siegelman J. Foveal drusen resorption one year after perifoveal laser photocoagulation. Ophthalmology 1991;98: 1379-83.

25 The Choroidal Neovascularization Prevention Trial Research Group. Laser treatment in eyes with large drusen. Short-term effects seen in a pilot randomized clinical trial. Ophthalmology 1998;105:11-23.

26 Miller H, Miller B, Ishibashi T, et al. Pathogenesis of laserinduced choroidal subretinal neovascularization. Invest Ophthalmol Vis Sci 1990;31:899-908.

27 Marshall J. Interactions between sensory cells, glial cells and the retinal pigment epithelium and their response to photocoagulation. Dev Ophthalmol 1981;2:308-17.

28 Wallow IH. Repair of the pigment epithelial barrier following photocoagulation. Arch Ophthalmol 1984;102:126-35.

29 Della NG, Wilson DJ, Klein ML. Clinical and pathologic effects of grid macular laser in aged primate eyes containing drusen. Invest Ophthalmol Vis Sci 1997;38 (suppl 4):s18.

30 Campochiaro PA, Hackett SF, Vinores SA, et al. Plateletderived growth factor is an autocrine growth stimulator in retinal pigment epithelial cells. F Cell Sci 1994;107:245969.

31 Yamamoto C, Ogata N, Yi X, et al. Immunolocalization of basic fibroblast growth factor during wound repair in rat retina after laser photocoagulation. Graefes Arch Clin Exp Ophthalmol 1996;11:695-702.

32 Duvall J, Tso MOM. Cellular mechanisms of resolution of drusen after laser photocoagulation: an experimental study. Arch Ophthalmol 1985;103:694-703.

33 Guymer RH, Hageman GS, Bird AC. Choroidal endothelial cell processes into Bruch's membrane. Invest Ophthalmol Vis Sci 1997;38 (suppl 4):s353. 